\title{
FORMULATIONS MIXTES AUGMENTÉES ET APPLICATIONS
}

\author{
BoujemâA Achchab ${ }^{1}$ et Abdellatif Agouzal ${ }^{1}$
}

\begin{abstract}
We propose and analyse a abstract framework for augmented mixed formulations. We give a priori error estimate in the general case: conforming and nonconforming approximations with or without numerical integration. Finally, a posteriori error estimator is given. An example of stabilized formulation for Stokes problem is analysed.
\end{abstract}

Résumé. On propose un cadre abstrait assez général, de formulations mixtes augmentées conformes ou non conformes, avec ou sans intégration numérique ; on fait l'analyse d'erreur a priori, et on propose des estimations d'erreur a posteriori pour ce genre de formulation, sans condition de compatibilité sur les espaces discrets. On traite un exemple de formulation stabilisée du problème de Stokes.

AMS Subject Classification. 65N30.

Reçu : 9 juin 1997. Révisé : 30 juin 1998.

\section{INTRODUCTION}

Plusieurs problèmes aux limites sont discrétisés par des formulations mixtes augmentées [6-8,11]. On distingue deux catégories: les formulations symétriques et les formulations non symétriques. La deuxième a l'avantage d'assurer que le problème discret est bien posé, sans contrainte, tandis que la première catégorie nécessite des contraintes, souvent difficiles à vérifier en pratique. Une tentative pour établir un cadre général a été développée par Barbosa et Hughes $[6,7]$, mais ce cadre n'est pas assez riche pour englober les cas d'approximation non-conforme et/ou avec intégration numérique. Nous allons développer un cadre général, permettant de traiter une large classe de problèmes ; en particulier les cas d'une approximation non conforme avec ou sans intégration numérique et nous allons nous intéresser aux estimateurs d'erreur de type hiérarchique introduits dans $[4,5]$ pour le cas elliptique non symétrique et non linéaire, et généralisés plus tard dans [1] aux formulations mixtes conformes ou non conformes et au cas de l'intégration numérique [9,12-14] ; ici la formulation augmentée sera exploitée pour définir le problème auxiliaire qui permet d'introduire l'indicateur, ou pour le problème intermédiaire posé sur les espaces les plus riches, et ce, dans le cas où ceux-ci ne sont pas compatibles [1]. Cet article est organisé comme suit : une première partie sera consacrée à présenter un cadre abstrait contenant les formulations mixtes augmentées et faisant le point sur les hypothèses que doivent vérifier les espaces discrets, ainsi que les opérateurs liés au problème traité ; on y présente ensuite les estimations d'erreur a priori pour les deux formulations symétriques et non symétriques.

Dans la deuxième partie, on exhibe dans le même cadre présenté, un estimateur hiérarchique équivalent à l'erreur exacte, et regroupant plusieurs cas comme le cas des formulations mixtes non conformes avec ou sans

\footnotetext{
Keywords and phrases. Mixed formulation, a posteriori error estimators.

1 Laboratoire d'Analyse Numérique, CNRS UMR 5585, Université Claude Bernard Lyon 1, 43 bd. du 11 Novembre 1918,69622

Villeurbanne Cedex, France. e-mail: Agouzal@iris.univ-lyon1.fr, achchab@lan1.univ-lyon1.fr
} 
intégration numérique. L'originalité de cet estimateur réside dans le fait que les espaces supplémentaires ne doivent pas être nécessairement compatibles comme c'est le cas de l'estimateur présenté dans [1], ce qui permet plus de souplesse dans le choix des espaces de discrétisations.

Dans la dernière partie, on présente un exemple d'application consacré au problème de Stokes stabilisé, où on présente un estimateur d'erreur pour l'élément $P_{1}^{+}-P_{1}$ enrichi en l'élément $P_{2}^{+}-P_{2}$.

\section{Cadre abstrait de formulations mixtes augmentées}

Dans ce paragraphe, nous allons développer un cadre abstrait général adapté aux formulations mixtes augmentées, nous considérerons le problème discret et nous donnerons enfin des estimations d'erreur a priori dans le cas d'approximation conforme ou non conforme avec ou sans intégration numérique.

\subsection{Problème continu}

Soient $X, X_{1}, M, M_{1}, H$ et $H_{1}$ des espaces de Hilbert vérifiant

$$
X \hookrightarrow X_{1}, M \hookrightarrow M_{1}, H \hookrightarrow H_{1}
$$

et $a(.,$.$) une forme bilinéaire continue sur X_{1} \times X_{1}, b(.,$.$) une forme bilinéaire continue sur M_{1} \times X_{1}$, et $l$ et $g$ deux formes linéaires définies et continues respectivement sur $X_{1}$ et $M_{1}$.

On considère le problème mixte abstrait suivant :

$$
(P)\left\{\begin{array}{l}
\text { trouver } u \in X, \lambda \in M \text { solution de : } \\
\forall v \in X, \quad a(u, v)+b(\lambda, v)=l(v) ; \\
\forall \mu \in M, \quad b(\mu, u)=g(\mu) .
\end{array}\right.
$$

Dans toute la suite, on suppose que le problème mixte $(P)$ admet une solution et une seule.

Pour obtenir une écriture compacte de notre problème, on introduit la forme bilinéaire $B(.,$.$) définie sur$ $\left(X_{1} \times M_{1}\right)^{2}$ par :

$$
\forall(u, \lambda),(v, \mu) \in X_{1} \times M_{1}, \quad B((u, \lambda),(v, \mu))=a(u, v)+b(\lambda, v)+b(\mu, u)
$$

et la forme linéaire $L$ définie sur $X_{1} \times M_{1}$ par :

$$
\forall(v, \mu) \in X_{1} \times M_{1}, \quad L((v, \mu))=l(v)+g(\mu) .
$$

Le problème mixte s'écrit alors sous la forme :

$$
(P)\left\{\begin{array}{l}
\text { trouver }(u, \lambda) \in X \times M, \\
B((u, \lambda),(v, \mu))=L(v, \mu), \quad \forall(v, \mu) \in X \times M .
\end{array}\right.
$$

Pour pouvoir développer les formulations mixtes augmentées, on suppose qu'il existe deux opérateurs linéaires $\mathcal{A} \in \mathcal{L}\left(X_{1}, H_{1}\right), \mathcal{B} \in \mathcal{L}\left(M_{1}, H_{1}\right)$, et un élément $f$ de $H$ tels que :

$$
\mathcal{A} u \in H, \mathcal{B} \lambda \in H,
$$

et

$$
\mathcal{A} u+\mathcal{B} \lambda=f \text { dans } H
$$

où $(u, \lambda)$ est la solution du problème $(P)$. 
Exemples. Nous allons donner quelques exemples de formulations mixtes vérifiant nos hypothèses. Pour cela, nous avons besoin de quelques notations utiles : soit $\Omega$ un ouvert polygonal de $\mathbb{R}^{d}(d \leq 3)$; dans tous les exemples considérés, $f \in L^{2}(\Omega)$ et $\underline{f} \in\left(L^{2}(\Omega)\right)^{d}$.

Exemple 1. Formulation primale des équations de Stokes

On considère le problème de Stokes :

$$
\begin{cases}-\Delta \underline{u}+\nabla p=\underline{f} & \text { dans } \Omega \\ \operatorname{div}(\underline{u})=0 & \text { dans } \Omega \\ \underline{u}=0 & \text { sur } \partial \Omega\end{cases}
$$

La formulation mixte correspondante est :

$$
\begin{cases}\underline{u} \in X, p \in M & \\ a(\underline{u}, \underline{v})+b(\underline{v}, p)=\langle\underline{f}, v\rangle_{0, \Omega}, & \forall v \in X, \\ b(\underline{u}, q)=0, & \forall q \in M .\end{cases}
$$

où

et

$$
\begin{gathered}
X=\left(H_{0}^{1}(\Omega)\right)^{2}, M=L_{0}^{2}(\Omega), \\
a(\underline{u}, \underline{v})=\int_{\Omega} \nabla \underline{u} . \nabla \underline{v} \mathrm{~d} x,
\end{gathered}
$$

$$
b(\underline{v}, p)=-\int_{\Omega} p \operatorname{div} \underline{v} \mathrm{~d} x .
$$

Soit $\mathcal{T}_{h}$ une famille de triangulations régulières de $\Omega$. On pose :

$$
X_{1}=X, \quad M_{1}=M,
$$

et

Enfin, on introduit les opérateurs :

$$
H=\prod_{K \in \mathcal{T}_{h}} L^{2}(K), \quad H_{1}=\prod_{K \in \mathcal{T}_{h}} H^{-1}(K) .
$$

$$
\begin{aligned}
\mathcal{A}: X & \longrightarrow H_{1}, \\
\underline{v} & \longmapsto \sum_{K \in T_{h}}\left(-\left.\Delta \underline{v}\right|_{K}\right),
\end{aligned}
$$

et

$$
\begin{aligned}
\mathcal{B}: M & \longrightarrow H_{1}, \\
q & \longmapsto \sum_{K \in T_{h}}\left(\nabla\left(\left.q\right|_{K}\right)\right) .
\end{aligned}
$$

On a :

$$
\mathcal{A} \underline{u}+\mathcal{B} p=f, \text { dans } H_{1} .
$$

Si de plus $\Omega$ est convexe, puisque $\underline{f} \in\left(L^{2}(\Omega)\right)^{d}$ on a :

$$
\mathcal{A} \underline{u} \in H \text { et } \quad \mathcal{B} p \in H .
$$


Exemple 2. Formulation duale des équations de Stokes

En écrivant les équations de Stokes sous la forme :

$$
\begin{cases}\underline{\sigma}-\nabla \underline{u}=0 & \operatorname{dans} \Omega \\ \operatorname{div} \underline{u}=0 & \operatorname{dans} \Omega \\ -\operatorname{div} \underline{\sigma}+\nabla p=\underline{f} & \operatorname{dans} \Omega \\ \underline{u}=0 & \text { sur } \partial \Omega .\end{cases}
$$

La formulation mixte s'écrit :

$$
\begin{cases}(\underline{\sigma}, \underline{p}) \in X, \underline{u} \in M & \\ a((\underline{\sigma}, \underline{p}),(\underline{\tau}, \underline{q}))+b((\underline{\tau}, \underline{q}), \underline{u}))=0 & \forall(\underline{\tau}, \underline{q}) \in X \\ b((\underline{\sigma}, \underline{p}), \underline{v}))=(\underline{f}, \underline{v})_{0, \Omega} & \forall \underline{v} \in M\end{cases}
$$

où

et

$$
\begin{gathered}
a((\underline{\sigma}, \underline{p}),(\underline{\tau}, \underline{q}))=(\underline{\sigma}: \underline{\tau})_{0, \Omega} \\
b((\underline{\tau}, \underline{p}), \underline{v}))=\langle\nabla \underline{\tau}: \nabla \underline{v}\rangle_{0, \Omega}-\langle\operatorname{div} \underline{v}, \underline{p}\rangle_{0, \Omega}
\end{gathered}
$$

$$
X=\left(L^{2}(\Omega)\right)^{4} \times L_{0}^{2}(\Omega), \quad M=\left(H_{0}^{1}(\Omega)\right)^{2} .
$$

On pose :

$$
H=\prod_{K \in \mathcal{T}_{h}}\left(L^{2}(K)\right)^{2} \quad \text { et } \quad H_{1}=\prod_{K \in \mathcal{T}_{h}}\left(H^{-1}(K)\right)^{2} .
$$

Enfin, on introduit les opérateurs :

$$
\begin{aligned}
\mathcal{A}: X & \longrightarrow H_{1} \\
(\underline{\sigma}, p) & \longmapsto-\operatorname{div} \underline{\sigma}+\nabla p,
\end{aligned}
$$

et

$$
\begin{aligned}
\mathcal{B}: M & \longrightarrow H_{1} \\
\underline{u} & \longmapsto \mathcal{B} \underline{u}=\underline{0} .
\end{aligned}
$$

Il est facile de vérifier :

$$
\mathcal{A}(\underline{\sigma}, p)=\underline{f}, \text { dans } H_{1}
$$

et

$$
\mathcal{A}(\underline{\sigma}, p) \in H \text {. }
$$

\section{Remarques.}

1. À partir de ces deux exemples, il est évident que l'hypothèse (2) est en général une hypothèse de régularité sur la solution $(u, \lambda)$ du problème $(P)$.

2. Soit $R$ une forme bilinéaire définie sur $H \times H$, on pose

$$
\begin{aligned}
& \bar{X}=\{u \in X, \mathcal{A} u \in H\}, \\
& \bar{M}=\{\mu \in M, \mathcal{B} \mu \in H\} .
\end{aligned}
$$


La solution $(u, \lambda)$ du problème $(P)$ est solution du problème :

$$
\left(P_{\delta}^{\prime}\right)\left\{\begin{array}{l}
(\bar{u}, \bar{\lambda}) \in \bar{X} \times \bar{M}, \\
\forall(\bar{v}, \bar{\mu}) \in \bar{X} \times \bar{M}, B((\bar{u}, \bar{\lambda}),(\bar{v}, \bar{\mu}))+R(\mathcal{A} \bar{u}+\mathcal{B} \bar{\lambda}, \mathcal{A} \bar{v}-\delta \mathcal{B} \bar{\mu})=L((\bar{u}, \bar{\lambda}))+R(f, \mathcal{A} \bar{v}-\delta \mathcal{B} \bar{\mu}),
\end{array}\right.
$$

où $\delta$ est un réel.

Remarquons de plus que si

$$
\left\|\left|(v, \mu)\left\|\left.\right|^{2}=\right\| v\left\|_{X}^{2}+\right\| \mathcal{B} \mu \|_{H}^{2}\right.\right.
$$

est une norme sur $\bar{X} \times \bar{M}$, alors le problème $\left(P_{\delta}^{\prime}\right)(\delta>0)$ admet au plus une solution.

C'est une idée similaire qui sera exploitée pour développer les formulations mixtes dites augmentées ou stabilisées.

\subsection{Problèmes discrets}

Soient $X_{h}$ et $M_{h}$ deux espaces de dimension finie vérifiant :

$$
X_{h} \hookrightarrow X_{1} ; M_{h} \hookrightarrow M_{1}
$$

et

$$
\mathcal{A} X_{h} \hookrightarrow H ; \mathcal{B} M_{h} \hookrightarrow H .
$$

Dans toute la suite, on désigne par $c, c_{0}, c_{1}, \ldots, \widehat{c}_{0}, \widehat{c}_{1}, \ldots$, diverses constantes génériques indépendantes de la dimension des espaces $X_{h}$ et $M_{h}$.

Soit $\langle., .\rangle_{h}$ une forme bilinéaire semi-définie positive sur $H \times H$. On introduit la semi-norme $\||\cdot \||$ définie sur $X_{1} \times \bar{M}_{1}$ par :

où

$$
\left\|\left.\left|(u, \lambda)\left\|\left.\right|^{2}=\right\| u \|_{X_{1}}^{2}+\right| \mathcal{B} \lambda\right|_{h} ^{2}\right.
$$

$$
\bar{M}_{1}=\left\{\mu \in M_{1}, \mathcal{B} \mu \in H\right\}
$$

et

$$
|\mathcal{B} \lambda|_{h}^{2}=\langle\mathcal{B} \lambda, \mathcal{B} \lambda\rangle_{h}
$$

Pour considérer un cadre assez général de discrétisation de notre problème mixte, cadre regroupant le cas d'une approximation conforme ou non, avec ou sans intégration numérique, nous allons introduire diverses formes linéaires ou bilinéaires nécessaires pour l'écriture du problème discret.

On considère les formes bilinéaires $a_{h}(.,$.$) et b_{h}(.,$.$) définies respectivement sur X_{h} \times X_{h}$ et $M_{h} \times X_{h}$, et les formes linéaires $l_{h}$ et $g_{h}$ définies et continues respectivement sur $X_{h}$ et $M_{h}$.

On suppose que :

$$
\begin{aligned}
a_{h}\left(v_{h}, v_{h}\right) & \geq c_{0}\left\|v_{h}\right\|_{X_{1}}^{2}, & & \forall v_{h} \in X_{h} \\
\left\langle\mathcal{A} v_{h}, \mathcal{A} v_{h}\right\rangle_{h} & \leq \gamma_{1}^{2}\left\|v_{h}\right\|_{X_{1}}^{2}, & & \forall v_{h} \in X_{h} \\
\frac{c_{0}}{2} & \geq \gamma_{1}^{2} & &
\end{aligned}
$$

avec $\gamma_{1}$ une constante indépendante de la dimension des espaces $X_{h}$ et $M_{h}$.

Remarques. L'hypothèse $(8)$ est facile à vérifier, quitte à multiplier la forme bilinéaire $\langle\mathcal{A} \text {., } \mathcal{A} .\rangle_{h}$ par une constante positive assez petite.

Pour avoir une écriture compacte du problème discret stabilisé, on introduit la forme bilinéaire $B_{\alpha, h}(.,$. 
définie sur $X_{h} \times M_{h}$ par :

$$
\begin{aligned}
\text { pour tout }\left(u_{h}, \lambda_{h}\right) \in & X_{h} \times M_{h},\left(v_{h}, \mu_{h}\right) \in X_{h} \times M_{h}, \\
B_{\alpha, h}\left(\left(u_{h}, \lambda_{h}\right),\left(v_{h}, \mu_{h}\right)\right)= & a_{h}\left(u_{h}, v_{h}\right)+b_{h}\left(\lambda_{h}, v_{h}\right)+b_{h}\left(\mu_{h}, u_{h}\right) \\
& +\alpha\left\langle\mathcal{A} u_{h}+\mathcal{B} \lambda_{h}, \mathcal{A} v_{h}-\alpha \mathcal{B} \mu_{h}\right\rangle_{h},
\end{aligned}
$$

et la forme linéaire $L_{\alpha, h}$ définie sur $X_{h} \times M_{h}$ par :

$$
\begin{aligned}
\text { pour tout } \mu_{h} & \in X_{h} \times M_{h} \\
L_{\alpha, h}\left(\left(v_{h}, \mu_{h}\right)\right) & =l_{h}\left(v_{h}\right)+g_{h}\left(\mu_{h}\right)+\alpha\left\langle f, \mathcal{A} v_{h}-\alpha \mathcal{B} \mu_{h}\right\rangle_{h}
\end{aligned}
$$

où $\alpha=-1,0,1$.

Nous considérons les deux formulations augmentées suivantes :

- formulation symétrique

$$
\left(P_{h}^{-}\right)\left\{\begin{array}{l}
\text { trouver } u_{h} \in X_{h}, \lambda_{h} \in M_{h}, \\
B_{-1, h}\left(\left(u_{h}, \lambda_{h}\right),\left(v_{h}, \mu_{h}\right)\right)=L_{-1, h}\left(\left(v_{h}, \mu_{h}\right)\right), \quad \forall\left(v_{h}, \mu_{h}\right) \in X_{h} \times M_{h},
\end{array}\right.
$$

- formulation non symétrique

$$
\left(P_{h}^{+}\right)\left\{\begin{array}{l}
\text { trouver } u_{h} \in X_{h}, \lambda_{h} \in M_{h}, \\
B_{1, h}\left(\left(u_{h}, \lambda_{h}\right),\left(v_{h}, \mu_{h}\right)\right)=L_{1, h}\left(\left(v_{h}, \mu_{h}\right)\right), \quad \forall\left(v_{h}, \mu_{h}\right) \in X_{h} \times M_{h} .
\end{array}\right.
$$

On peut aussi écrire les deux problèmes $\left(P_{h}^{-}\right)$et $\left(P_{h}^{+}\right)$sous les formes équivalentes suivantes :

- formulation symétrique

$$
\left(P_{h}^{-}\right)\left\{\begin{array}{l}
\text { trouver } u_{h} \in X_{h}, \lambda_{h} \in M_{h} \text { solution de : } \\
\forall v_{h} \in X_{h}, a_{-1, h}\left(u_{h}, v_{h}\right)+b_{-1, h}\left(\lambda_{h}, v_{h}\right)=l_{-1, h}\left(v_{h}\right) \\
\forall \mu_{h} \in M_{h}, b_{-1, h}\left(\mu_{h}, u_{h}\right)+c_{h}\left(\lambda_{h}, \mu_{h}\right)=g_{-1, h}\left(\mu_{h}\right)
\end{array}\right.
$$

- formulation non symétrique

$$
\left(P_{h}^{+}\right)\left\{\begin{array}{l}
\text { trouver } u_{h} \in X_{h}, \lambda_{h} \in M_{h} \text { solution de }: \\
\forall v_{h} \in X_{h}, a_{1, h}\left(u_{h}, v_{h}\right)+b_{1, h}\left(\lambda_{h}, v_{h}\right)=l_{1, h}\left(v_{h}\right) \\
\forall \mu_{h} \in M_{h}, b_{1, h}\left(\mu_{h}, u_{h}\right)+c_{h}\left(\lambda_{h}, \mu_{h}\right)=g_{1, h}\left(\mu_{h}\right)
\end{array}\right.
$$

où

$$
\begin{aligned}
& a_{\alpha, h}\left(u_{h}, v_{h}\right)=a_{h}\left(u_{h}, v_{h}\right)+\alpha\left\langle\mathcal{A} u_{h}, \mathcal{A} v_{h}\right\rangle_{h}, \\
& b_{\alpha, h}\left(\lambda_{h}, v_{h}\right)=b_{h}\left(\lambda_{h}, v_{h}\right)+\alpha\left\langle\mathcal{B} \lambda_{h}, \mathcal{A} v_{h}\right\rangle_{h}
\end{aligned}
$$

et

$$
c_{h}\left(\lambda_{h}, \mu_{h}\right)=-\left\langle\mathcal{B} \lambda_{h}, \mathcal{B} \mu_{h}\right\rangle_{h}
$$

\subsection{Existence et unicité des solutions des problèmes discrets}

Dans ce paragraphe, nous allons établir l'existence et l'unicité du problème discret, ainsi que certains résultats très utiles pour établir des estimations d'erreur a priori et a posteriori. Tout d'abord pour le cas de la formulation 
symétrique, on a le résultat suivant :

Lemme 1. Si les conditions (6-8) sont vérifiées, alors la forme bilinéaire symétrique $B_{-1, h}(.,$.$) satisfait la$ condition inf-sup suivante :

$$
\inf _{\left(u_{h}, \lambda_{h}\right) \in X_{h} \times M_{h}} \sup _{\left(v_{h}, \mu_{h}\right) \in X_{h} \times M_{h}} \frac{B_{-1, h}\left(\left(u_{h}, \lambda_{h}\right),\left(v_{h}, \mu_{h}\right)\right)}{\left\|\left|( v _ { h } , \mu _ { h } ) \left\|\left|\left\|\left|\left(u_{h}, \lambda_{h}\right) \|\right|\right.\right.\right.\right.\right.} \geq C>0 .
$$

Preuve. Soit $\left(u_{h}, \lambda_{h}\right) \in X_{h} \times M_{h}$. On a

$$
\begin{aligned}
B_{-1, h}\left(\left(u_{h}, \lambda_{h}\right),\left(u_{h},-\lambda_{h}\right)\right) & =a_{h}\left(u_{h}, u_{h}\right)-\left\langle\mathcal{A} u_{h}+\mathcal{B} \lambda_{h}, \mathcal{A} u_{h}-\mathcal{B} \lambda_{h}\right\rangle_{h} \\
& =a_{h}\left(u_{h}, u_{h}\right)-\left(\left|\mathcal{A} u_{h}\right|_{h}^{2}-\left|\mathcal{B} \lambda_{h}\right|_{h}^{2}\right) \\
& \geq c_{0}\left\|u_{h}\right\|_{X_{1}}^{2}-\gamma_{1}^{2}\left\|u_{h}\right\|_{X_{1}}^{2}+\left|\mathcal{B} \lambda_{h}\right|_{h}^{2} \\
& =\left(c_{0}-\gamma_{1}^{2}\right)\left\|u_{h}\right\|_{X_{1}}^{2}+\left|\mathcal{B} \lambda_{h}\right|_{h}^{2} \\
& \geq \widetilde{c}_{0}\left(\left\|u_{h}\right\|_{X_{1}}^{2}+\left|\mathcal{B} \lambda_{h}\right|_{h}^{2}\right) \quad\left(\operatorname{car} \gamma_{1}^{2} \leq \frac{c_{0}}{2}\right)
\end{aligned}
$$

avec $\widetilde{c}_{0}=\min \left\{c_{0} / 2,1\right\}$, on a donc le résultat avec $C=\widetilde{c}_{0}$.

Pour le cas non symétrique, on a :

Lemme 2. Si les conditions (6-7) sont satisfaites, alors la forme bilinéaire non symétrique $B_{1, h}(.,$.$) satisfait$ la condition inf-sup suivante :

$$
\inf _{\left(u_{h}, \lambda_{h}\right) \in X_{h} \times M_{h}} \sup _{\left(v_{h}, \mu_{h}\right) \in X_{h} \times M_{h}} \frac{B_{1, h}\left(\left(u_{h}, \lambda_{h}\right),\left(v_{h}, \mu_{h}\right)\right)}{\left\|\left|( v _ { h } , \mu _ { h } ) \left\|\left|\left\|\left|\left(u_{h}, \lambda_{h}\right) \|\right|\right.\right.\right.\right.\right.} \geq C>0 .
$$

Preuve. Soit $\left(u_{h}, \lambda_{h}\right) \in X_{h} \times M_{h}$. On a

$$
\begin{aligned}
B_{1, h}\left(\left(u_{h}, \lambda_{h}\right),\left(u_{h},-\lambda_{h}\right)\right) & =a_{h}\left(u_{h}, u_{h}\right)+\left\langle\mathcal{A} u_{h}+\mathcal{B} \lambda_{h}, \mathcal{A} u_{h}+\mathcal{B} \lambda_{h}\right\rangle_{h} \\
& =a_{h}\left(u_{h}, u_{h}\right)+\left|\mathcal{A} u_{h}+\mathcal{B} \lambda_{h}\right|_{h}^{2} \\
& \geq c_{0}\left\|u_{h}\right\|_{X_{1}}^{2}+\left(\left|\mathcal{A} u_{h}\right|_{h}^{2}+\left|\mathcal{B} \lambda_{h}\right|_{h}^{2}-\left(\kappa_{0}\left|\mathcal{A} u_{h}\right|_{h}^{2}+\frac{1}{\kappa_{0}}\left|\mathcal{B} \lambda_{h}\right|_{h}^{2}\right)\right)
\end{aligned}
$$

On choisit $\kappa_{0}=1+c_{0} / 2 \gamma_{1}^{2}$; il s'ensuit que :

$$
\begin{aligned}
B_{1, h}\left(\left(u_{h}, \lambda_{h}\right),\left(u_{h},-\lambda_{h}\right)\right) & \geq c_{0}\left\|u_{h}\right\|_{X_{1}}^{2}+\left(1-\kappa_{0}\right)\left|\mathcal{A} u_{h}\right|_{h}^{2}+\left(1-\frac{1}{\kappa_{0}}\right)\left|\mathcal{B} \lambda_{h}\right|_{h}^{2} \\
& \geq c_{0}\left\|u_{h}\right\|_{X_{1}}^{2}-\frac{c_{0}}{2 \gamma_{1}^{2}}\left|\mathcal{A} u_{h}\right|_{h}^{2}+\frac{c_{0}}{2 \gamma_{1}^{2}+c_{0}}\left|\mathcal{B} \lambda_{h}\right|_{h}^{2} \\
& \geq \frac{c_{0}}{2}\left\|u_{h}\right\|_{X_{1}}^{2}+\frac{c_{0}}{2 \gamma_{1}^{2}+c_{0}}\left|\mathcal{B} \lambda_{h}\right|_{h}^{2} \quad\left[\mathrm{~d}^{\prime} \text { après }(7)\right] \\
& \geq \widetilde{c}_{0}\left(\left\|u_{h}\right\|_{X_{1}}^{2}+\left|\mathcal{B} \lambda_{h}\right|_{h}^{2}\right)
\end{aligned}
$$

où $\widetilde{c}_{0}=\min \left\{\frac{c_{0}}{2}, \frac{c_{0}}{2 \gamma_{1}^{2}+c_{0}}\right\}$. On en déduit le résultat désiré.

Remarque. Dans le cas non symétrique, l'hypothèse (8) n'est pas nécessaire.

On déduit facilement des deux lemmes précédents le :

Corollaire 1. Si $\| \mid$. $\| \mid$ est une norme sur $X_{1} \times M_{1}$, et sous les hypothèses du lemme 1 (resp. lemme 2) ; le problème $\left(P_{h}^{-}\right)$(resp. $\left.\left(P_{h}^{+}\right)\right)$admet une solution et une seule. 


\subsection{Estimations d'erreur a priori}

Concernant les estimations d'erreur a priori, on a les deux théorèmes suivants dont les démonstrations sont similaires :

Théorème 1. Soient $U=(u, \lambda)$ la solution du problème mixte $(P)$ et $U_{h}=\left(u_{h}, \lambda_{h}\right)$ la solution du problème mixte discret $\left(P_{h}^{-}\right)$. On suppose que $(u, \lambda) \in \bar{X} \times \bar{M}$ et qu'il existe un élément $f$ de $H$ tel que (3) soit vérifiée. Alors, sous les hypothèses (2) et (6-8), on a les estimations a priori suivantes :

$$
\begin{aligned}
\left\|U-U_{h}\right\| \leq & C \inf _{V_{h}=\left(v_{h}, \mu_{h}\right) \in X_{h} \times M_{h}}\left\{\left\|\left|U-V_{h} \|\right|+\left|\mathcal{A}\left(u-v_{h}\right)\right|_{h}\right.\right. \\
& \left.+\quad \sup _{W_{h} \in X_{h} \times M_{h}} \frac{B_{0, h}\left(V_{h}, W_{h}\right)-L_{0, h}\left(W_{h}\right)}{\left\|W_{h}\right\| \mid}\right\} .
\end{aligned}
$$

Théorème 2. Soient $U=(u, \lambda)$ la solution du problème mixte $(P)$ et $U_{h}=\left(u_{h}, \lambda_{h}\right)$ la solution du problème mixte discret $\left(P_{h}^{+}\right)$. On suppose que $(u, \lambda) \in \bar{X} \times \bar{M}$ et qu'il existe un élément $f$ de $H$ tel que (3) soit vérifiée. Alors, sous les hypothèses (2) et (6-7), on a les estimations a priori suivantes :

$$
\begin{aligned}
\left\|U-U_{h}\right\| \leq & C \inf _{V_{h}=\left(v_{h}, \mu_{h}\right) \in X_{h} \times M_{h}}\left\{\left\|\left|U-V_{h} \|\right|+\left|\mathcal{A}\left(u-v_{h}\right)\right|_{h}\right.\right. \\
& \left.+\quad \sup _{W_{h} \in X_{h} \times M_{h}} \frac{B_{0, h}\left(V_{h}, W_{h}\right)-L_{0, h}\left(W_{h}\right)}{\left\|W_{h}\right\| \mid}\right\} .
\end{aligned}
$$

Preuve. D'une part, pour tout $V_{h}=\left(v_{h}, \mu_{h}\right) \in X_{h} \times M_{h}$ et pour $\alpha=-1,1$, on a :

et (lemme 1 ou 2)

$$
\left\|\left|U-U_{h}\left\|\left|\leq\left\|\left|U-V_{h}\left\|\left|+\left\|\left|V_{h}-U_{h} \|\right|,\right.\right.\right.\right.\right.\right.\right.\right.\right.
$$

$$
\left\|\left|V_{h}-U_{h} \|\right| \leq C \sup _{W_{h} \in X_{h} \times M_{h}} \frac{B_{\alpha, h}\left(V_{h}-U_{h}, W_{h}\right)}{\left\|\left|W_{h} \|\right|\right.} .\right.
$$

D'autre part, pour tout $W_{h}=\left(w_{h}, \delta_{h}\right) \in X_{h} \times M_{h}$, on a :

$$
\begin{aligned}
B_{\alpha, h}\left(V_{h}-U_{h}, W_{h}\right)= & B_{\alpha, h}\left(V_{h}, W_{h}\right)-L_{\alpha, h}\left(W_{h}\right) \\
= & B_{0, h}\left(V_{h}, W_{h}\right)-L_{0, h}\left(W_{h}\right) \\
& +\alpha\left\langle\mathcal{A}\left(v_{h}-u\right)+\mathcal{B}\left(\mu_{h}-\lambda\right), \mathcal{A} w_{h}-\alpha \mathcal{B} \delta_{h}\right\rangle_{h} \\
\leq & B_{0, h}\left(V_{h}, W_{h}\right)-L_{0, h}\left(W_{h}\right) \\
& +\left\{\left|\mathcal{A}\left(v_{h}-u\right)\right|_{h}+\left|\mathcal{B}\left(\mu_{h}-\lambda\right)\right|_{h}\right\}\left\{\gamma_{1}\left\|w_{h}\right\|_{X_{1}}+\left|\mathcal{B} \delta_{h}\right|_{h}\right\}
\end{aligned}
$$

Par suite, il s'ensuit :

$$
\begin{array}{r}
\left\|\left|U-U_{h} \|\right| \leq \operatorname{linf}_{V_{h}=\left(v_{h}, \mu_{h}\right) \in X_{h} \times M_{h}}\left\{\left\|\left|U-V_{h} \|\right|+\left|\mathcal{A}\left(u-v_{h}\right)\right|_{h}\right.\right.\right. \\
\left.+\sup _{W_{h} \in X_{h} \times M_{h}} \frac{B_{0, h}\left(V_{h}, W_{h}\right)-L_{0, h}\left(W_{h}\right)}{\left\|W_{h}\right\| \mid}\right\} .
\end{array}
$$


Dans le cas d'une approximation conforme sans intégration numérique, les théorèmes 1 ou 2, nous permettent d'obtenir le

Corollaire 2. On suppose que $X_{1}=X$ et $M_{1}=M$, sous les hypothèses du théorème 1 ou le théorème 2, on $a$ :

$$
\begin{aligned}
\left\|\left|U-U_{h} \|\right| \leq\right. & C \inf _{\left(v_{h}, \mu_{h}\right) \in X_{h} \times M_{h}}\left\{\left\|u-v_{h}\right\|_{X}+\left\|\lambda-\mu_{h}\right\|_{M}+\left|\mathcal{A}\left(u-v_{h}\right)\right|_{h}\right. \\
& \left.+\left|\mathcal{B}\left(\lambda-\mu_{h}\right)\right|_{h}+\sup _{\chi_{h} \in M_{h}} \frac{b\left(\chi_{h}, v_{h}-u\right)}{\left|\mathcal{B} \chi_{h}\right|_{h}}\right\} .
\end{aligned}
$$

Preuve. Elle découle directement des estimations précédentes, en remarquant que :

$$
B\left(V_{h}, W_{h}\right)-L\left(W_{h}\right)=a\left(v_{h}-u, w_{h}\right)+b\left(\lambda-\mu_{h}, w_{h}\right)+b\left(\chi_{h}, v_{h}-u\right)
$$

Remarque. L'estimation du terme $\sup _{\chi_{h} \in M_{h}} \frac{b\left(\chi_{h}, v_{h}-u\right)}{\left|\mathcal{B} \chi_{h}\right|_{h}}$ est essentielle : elle dépend surtout du problème traité et des espaces de discrétisation utilisés. Cette estimation peut ne pas être optimale, dans certains cas (voir par exemple [3] dans le cas de l'h-p version en décomposition de domaines). Remarquons qu'une majoration "naïve" de type :

$$
\sup _{\chi_{h} \in M_{h}} \frac{b\left(\chi_{h}, v_{h}-u\right)}{\left|\mathcal{B} \chi_{h}\right|_{h}} \leq C\left|v_{h}-u\right|_{X} \cdot\left(\sup _{\chi_{h} \in M_{h}} \frac{\left|\chi_{h}\right|_{M}}{\left|\mathcal{B} \chi_{h}\right|_{h}}\right)
$$

n'est pas optimale en général, car $\sup _{\chi_{h} \in M_{h}} \frac{\left|\chi_{h}\right|_{M}}{\left|\mathcal{B} \chi_{h}\right|_{h}}$ dépend fortement des paramètres de discrétisation.

Dans le cas d'une approximation conforme avec intégration numérique, on a :

Corollaire 3. Sous les mêmes hypothèses que le corollaire précédent, on a :

$$
\begin{aligned}
\left\|\mid U-U_{h}\right\| \leq \leq & C \inf _{v_{h} \in X_{h}}\left\{\left\|u-v_{h}\right\|_{X}+\left|\mathcal{A}\left(u-v_{h}\right)\right|_{h}+\sup _{\chi_{h} \in M_{h}}\left(\frac{b\left(\chi_{h}, u-v_{h}\right)}{\left\|\chi_{h}\right\|_{M}}\right.\right. \\
& \left.\left.\quad+\frac{b_{h}\left(\chi_{h}, v_{h}\right)-b\left(\chi_{h}, v_{h}\right)}{\left\|\chi_{h}\right\|_{M}}\right)+\sup _{w_{h} \in X_{h}} \frac{a_{h}\left(v_{h}, w_{h}\right)-a\left(v_{h}, w_{h}\right)}{\left\|w_{h}\right\|_{X}}\right\} \\
& +\inf _{\mu_{h} \in X_{h}}\left\{\left|\lambda-\mu_{h}\right|_{M}+\sup _{w_{h} \in X_{h}}\left\{\frac{b_{h}\left(\mu_{h}, w_{h}\right)-b\left(\mu_{h}, w_{h}\right)}{\left\|w_{h}\right\|_{X}}\right\}\right\} \\
& \left.+\sup _{\chi_{h} \in M_{h}} \frac{g_{h}\left(\chi_{h}\right)-g\left(\chi_{h}\right)}{\left\|\chi_{h}\right\|_{M}}+\sup _{w_{h} \in X_{h}} \frac{l_{h}\left(w_{h}\right)-l\left(w_{h}\right)}{\left\|w_{h}\right\|_{M}}\right\} .
\end{aligned}
$$

Preuve. Pour tout $V_{h}=\left(v_{h}, \mu_{h}\right) \in X_{h} \times M_{h}$ et $W_{h}=\left(w_{h}, \chi_{h}\right) \in X_{h} \times M_{h}$ on a :

$$
\begin{aligned}
B_{0, h}\left(V_{h}, W_{h}\right)-L_{0, h}\left(W_{h}\right)= & B_{0, h}\left(V_{h}, W_{h}\right)-B\left(V_{h}, W_{h}\right)+L\left(W_{h}\right) \\
& -L_{0, h}\left(W_{h}\right)-B\left(U-V_{h}, W_{h}\right) .
\end{aligned}
$$

Or

$$
\begin{aligned}
B_{0, h}\left(V_{h}, W_{h}\right)-B\left(V_{h}, W_{h}\right)= & a_{h}\left(v_{h}, w_{h}\right)-a\left(v_{h}, w_{h}\right)+b_{h}\left(\mu_{h}, w_{h}\right)-b\left(\mu_{h}, w_{h}\right) \\
& +b_{h}\left(\chi_{h}, v_{h}\right)-b\left(\chi_{h}, v_{h}\right)+l\left(w_{h}\right)-l_{h}\left(w_{h}\right) \\
& +g\left(\chi_{h}\right)-g_{h}\left(\chi_{h}\right) .
\end{aligned}
$$


Par suite

$$
\begin{aligned}
\frac{B_{0, h}\left(V_{h}, W_{h}\right)-B\left(V_{h}, W_{h}\right)}{\left\|W_{h}\right\| \mid} \leq & \frac{a_{h}\left(v_{h}, w_{h}\right)-a\left(v_{h}, w_{h}\right)}{\left\|w_{h}\right\|_{X}}+\frac{b_{h}\left(\mu_{h}, w_{h}\right)-b\left(\mu_{h}, w_{h}\right)}{\left\|w_{h}\right\|_{X}} \\
& +\frac{b_{h}\left(\chi_{h}, v_{h}\right)-b\left(\chi_{h}, v_{h}\right)}{\left\|\chi_{h}\right\|_{X}}+\frac{l\left(w_{h}\right)-l_{h}\left(w_{h}\right)}{\left\|w_{h}\right\|_{X}} \\
& +\frac{g\left(\chi_{h}\right)-g_{h}\left(\chi_{h}\right)}{\left\|\chi_{h}\right\|_{X}} .
\end{aligned}
$$

En utilisant cette dernière inégalité et le même raisonnement pour traiter le terme $B\left(U-V_{h}, W_{h}\right)$, on obtient le résultat.

Sous les hypothèses de chacun des deux théorèmes et si l'on suppose en plus que la forme bilinéaire $b_{h}(.,$. vérifie :

$$
\forall \mu_{h} \in M_{h}, \quad \sup _{v_{h} \in X_{h}} \frac{b_{h}\left(\mu_{h}, v_{h}\right)}{\left\|v_{h}\right\|_{X_{1}}} \geq c_{4}\left\|\mu_{h}\right\|_{M_{1}}-c_{5}\left|\mathcal{B} \mu_{h}\right|_{h}
$$

alors, on peut avoir les mêmes estimations d'erreur a priori avec la norme \|\|$_{Q_{1}, h}$ définie par :

$$
\|(v, \mu)\|_{Q_{1}, h}^{2}=\|v\|_{X_{1}}^{2}+\left\|\mu_{h}\right\|_{M_{1}}^{2}+|\mathcal{B} \mu|_{h}^{2}, \quad \forall(v, \mu) \in X_{1} \times \bar{M}_{1} .
$$

Plus précisément, on a tout d'abord le théorème suivant dont la démonstration suit essentiellement celles des théorèmes 1 et 2 en la combinant avec des arguments développés par Barbosa et Hughes [6,7] :

Théorème 3. Sous les conditions (6-7) [resp. (6-8)], la forme bilinéaire symétrique $B_{-1, h}(.,$.$) [resp. non$ symétrique] vérifie :

$$
\inf _{\left(u_{h}, \lambda_{h}\right) \in X_{h} \times M_{h}} \sup _{\left(v_{h}, \mu_{h}\right) \in X_{h} \times M_{h}} \frac{B_{ \pm 1, h}\left(\left(u_{h}, \lambda_{h}\right),\left(v_{h}, \mu_{h}\right)\right)}{\left\|\left(v_{h}, \mu_{h}\right)\right\|_{Q_{1}, h}\left\|\left(u_{h}, \lambda_{h}\right)\right\|_{Q_{1}, h}} \geq C>0 .
$$

Cette hypothèse supplémentaire est très utile pour l'analyse du problème et surtout pour l'obtention des estimations d'erreur a priori. Le corollaire 2 devient dans ce cas :

Corollaire 4. On suppose que $X_{1}=X$ et $M_{1}=M$, sous les hypothèses du théorème 1 ou du théorème 2, on $a$ :

$$
\left\|U-U_{h}\right\|_{Q_{1}, h} \leq C \inf _{V_{h}=\left(v_{h}, \mu_{h}\right) \in X_{h} \times M_{h}}\left\{\left\|U-V_{h}\right\|_{Q_{1}, h}+\left|\mathcal{A}\left(u-v_{h}\right)\right|_{h}\right\} .
$$

\section{Remarques.}

- L'estimation du terme $\sup _{\chi_{h} \in M_{h}} \frac{b\left(v_{h}-u_{h}, \chi_{h}\right)}{\left|\mathcal{B} \chi_{h}\right|_{h}}$ qui constitue une difficulté dans le corollaire 2, est soulevée dans ce cas.

- Il est intéressant de connaître des conditions suffisantes permettant d'assurer (15). Franca-Hughes et Stenberg [11] ont donné une condition suffisante dans le cas de formulation mixte des équations de Stokes et de Navier-Stokes. Plus précisément, en utilisant les notations de l'exemple 2 du paragraphe 2.1, on a le

Lemme 3. Soit $\mathcal{T}_{h}$ une triangulation régulière de $\Omega$ en triangles, si :

$$
\left\{v_{h} \in\left(\mathcal{C}^{0}(\bar{\Omega})\right)^{2} ; \forall K \in \mathcal{T}_{h},\left.v_{h}\right|_{K} \in P_{d}(K)\right\} \subset X_{h} \subset\left(H_{0}^{1}(\Omega)\right)^{2}
$$

ou si $M_{h}$ vérifie :

$$
M_{h} \subset \mathcal{C}^{0}(\bar{\Omega}),
$$


alors

$$
\forall p_{h} \in M_{h}, \sup _{v_{h} \in X_{h}} \frac{\int_{\Omega} \operatorname{div} v_{h} p_{h} \mathrm{~d} x}{\left\|v_{h}\right\|_{1, \Omega}} \geq C_{1}\left\|p_{h}\right\|_{0, \Omega}-C_{2}\left(\sum_{K \in \mathcal{T}_{h}} h_{K}^{2}\left|p_{h}\right|_{1, K}^{2}\right)^{\frac{1}{2}} .
$$

Dans le cas d'une formulation hybride primale d'une équation elliptique, obtenue par dualisation d'une condition de Dirichlet, cette condition est toujours vérifiée (voir [6]).

\section{Estimations D'ERREur a POSTERIORI HiÉRARCHIQUES}

Dans cette partie, nous donnerons des estimations d'erreur a posteriori dans le cas d'approximation conforme ou non conforme, avec ou sans intégration numérique.

On garde les mêmes notations qu'au paragraphe précédent et on considère le problème mixte abstrait suivant :

$$
(P)\left\{\begin{array}{l}
\text { trouver } u \in X, \lambda \in M \text { solution de : } \\
\forall v \in X, \quad a(u, v)+b(\lambda, v)=l(v) \\
\forall \mu \in M, \quad b(\mu, u)=g(\mu)
\end{array}\right.
$$

conformément au paragraphe 2.1, on suppose qu'il existe un espace de Hilbert $H$, deux opérateurs linéaires $\mathcal{A} \in \mathcal{L}\left(X_{1}, H\right), \mathcal{B} \in \mathcal{L}\left(M_{1}, H\right)$, et un élément $f$ de $H$ tels que :

$$
\mathcal{A} u \in H, \quad \mathcal{B} \lambda \in H
$$

et

$$
\mathcal{A} u+\mathcal{B} \lambda=f \quad \text { dans } H,
$$

où $(u, \lambda)$ est la solution du problème mixte abstrait $(P)$.

Pour obtenir une approximation de $(u, \lambda)$, on considère le problème discret suivant :

$$
\left(P_{h}\right)\left\{\begin{array}{l}
\text { trouver } u_{h} \in X_{h}, \lambda_{h} \in M_{h} \text { solution de : } \\
\forall v_{h} \in X_{h}, a_{h}\left(u_{h}, v_{h}\right)+b_{h}\left(\lambda_{h}, v_{h}\right)=l_{h}\left(v_{h}\right) \\
\forall \mu_{h} \in M_{h}, b_{h}\left(\mu_{h}, u_{h}\right)=f_{h}\left(\mu_{h}\right)
\end{array}\right.
$$

On pose :

$$
\begin{gathered}
Q_{1}=X_{1} \times M_{1}, \quad Q=X \times M \\
Q_{h}=X_{h} \times M_{h},
\end{gathered}
$$

et on considère l'espace $\bar{Q}_{h}=\bar{X}_{h} \times \bar{M}_{h}$ de dimension finie contenant $Q_{h}$ et contenu dans $Q_{1}\left(\bar{Q}_{h} \hookrightarrow Q_{1}\right)$, avec $\bar{X}_{h}$, et $\bar{M}_{h}$ des espaces de Hilbert vérifiant :

$$
X_{h} \subset \bar{X}_{h} \subset X_{1} \text { et } M_{h} \subset \bar{M}_{h} \subset M_{1} \text {. }
$$

On considère la décomposition suivante :

$$
\left\{\begin{array}{c}
\bar{X}_{h}=X_{h} \oplus \widehat{X}_{h} \\
\bar{M}_{h}=M_{h} \oplus \widehat{M}_{h}
\end{array}\right.
$$

On pose :

$$
\widehat{Q}_{h}=\widehat{X}_{h} \times \widehat{M}_{h},
$$




$$
T_{h}=\left\{\mu_{h} \in M_{h} ; \forall v_{h} \in X_{h}, b_{h}\left(\mu_{h}, v_{h}\right)=0\right\}
$$

et

$$
\bar{T}_{h}=\left\{\mu_{h} \in \bar{M}_{h} ; \forall v_{h} \in \bar{X}_{h}, \bar{b}_{h}\left(\mu_{h}, v_{h}\right)=0\right\}
$$

où $\bar{b}_{h}$ est une forme bilinéaire définie et continue sur $\bar{X}_{h} \times \bar{M}_{h}$.

Il est facile de vérifier que :

$$
\bar{Q}_{h}=Q_{h} \oplus \widehat{Q}_{h}
$$

Enfin, on considère une forme bilinéaire $\bar{a}_{h}(.,$.$) définie sur \bar{X}_{h} \times \bar{X}_{h}$, et des formes linéaires continues $\bar{l}_{h}, \bar{g}_{h}$ respectivement sur $\bar{X}_{h}$ et $\bar{M}_{h}$, et l'on suppose que :

$$
\begin{aligned}
\forall v_{h} \in \widetilde{X}_{h}, \quad \forall w_{h} \in \widetilde{X}_{h}, \quad \widetilde{a}_{h}\left(v_{h}, w_{h}\right) & \leq \widetilde{c}_{1}\left\|v_{h}\right\|_{X_{1}}\left\|w_{h}\right\|_{X_{1}}, \\
\forall v_{h} \in \widetilde{X}_{h}, \quad \forall \mu_{h} \in \widetilde{M}_{h}, \quad \widetilde{b}_{h}\left(\mu_{h}, v_{h}\right) & \leq \widetilde{c}_{2}\left\|\mu_{h}\right\|_{M_{1}}\left\|v_{h}\right\|_{X_{1}}, \\
\inf _{\mu_{h} \in \widetilde{M}_{h}} \sup _{v_{h} \in \widetilde{X}_{h}} \frac{\widetilde{b}_{h}\left(\mu_{h}, v_{h}\right)}{\left\|\mu_{h}\right\|_{M_{1}}\left\|v_{h}\right\|_{X_{1}}} & \geq \widetilde{\beta}_{1}>0, \\
\forall v_{h} \in \widetilde{T}_{h}, \quad \widetilde{a}_{h}\left(v_{h}, v_{h}\right) & \geq \widetilde{\alpha}\left\|v_{h}\right\|_{X} \quad \operatorname{avec}(\widetilde{\alpha}>0)
\end{aligned}
$$

où

$$
\begin{aligned}
\widetilde{a}_{h}(., .) & =a_{h}(., .), \bar{a}_{h}(., .) ; \\
\widetilde{b}_{h}(., .) & =b_{h}(., .), \bar{b}_{h}(., .) ; \\
\widetilde{X}_{h} & =X_{h}, \bar{X}_{h} ; \\
\widetilde{M}_{h} & =M_{h}, \bar{M}_{h} ; \\
\widetilde{T}_{h} & =T_{h}, \bar{T}_{h}
\end{aligned}
$$

où $\widetilde{\beta}_{1}, \tilde{\alpha}, \widetilde{c}_{1}, \widetilde{c}_{2}$ sont des constantes strictement positives indépendantes des dimensions des espaces (dimensions finies).

On est en mesure de définir alors le problème intermédiaire suivant :

$$
\left(\bar{P}_{h}\right)\left\{\begin{array}{l}
\text { trouver } \bar{u}_{h} \in \bar{X}_{h}, \bar{\lambda}_{h} \in \bar{M}_{h} \text { solution de : } \\
\forall \bar{v}_{h} \in \bar{X}_{h}, \bar{a}_{h}\left(\bar{u}_{h}, \bar{v}_{h}\right)+\bar{b}_{h}\left(\bar{\lambda}_{h}, \bar{v}_{h}\right)=\bar{l}_{h}\left(\bar{v}_{h}\right), \\
\forall \bar{\mu}_{h} \in \bar{M}_{h}, \bar{b}_{h}\left(\bar{\mu}_{h}, \bar{u}_{h}\right)=\bar{g}_{h}\left(\bar{\mu}_{h}\right) .
\end{array}\right.
$$

En utilisant le théorie de Babuska-Brezzi [8], il est facile de montrer la

Proposition 1. Si les conditions (21-24) sont vérifiées, alors les problèmes $\left(P_{h}\right)$ et $\left(\bar{P}_{h}\right)$ sont bien posés.

Pour pouvoir définir l'estimateur a posteriori, nous avons besoin d'introduire un problème auxiliaire défini sur $\widehat{Q}_{h}$; pour cela nous avons besoin d'introduire certaines notations.

On considère alors deux formes bilinéaires $\widehat{a}_{h}(.,),. \widehat{b}_{h}(.,$.$) définies respectivement sur \widehat{Q}_{h}$, et $\widehat{M}_{h} \times \widehat{X}_{h}$, et deux formes linéaires $\widehat{l}_{h}$ et $\widehat{g}_{h}$ définies et continues respectivement sur $\widehat{X}_{h}$ et $\widehat{M}_{h}$. 
Dans toute la suite de ce paragraphe, on suppose que :

$$
\begin{array}{rlrl}
\forall v_{h} \in \widehat{X}_{h}, \forall w_{h} & \in \widehat{X}_{h}, & \widehat{a}_{h}\left(v_{h}, w_{h}\right) & \leq \widehat{c}_{1}\left\|v_{h}\right\|_{X_{1}}\left\|w_{h}\right\|_{X_{1}} \\
\forall v_{h} \in \widehat{X}_{h}, \forall \mu_{h} & \in \widehat{M}_{h}, & \widehat{b}_{h}\left(\mu_{h}, v_{h}\right) & \leq \widehat{c}_{2}\left\|\mu_{h}\right\|_{M_{1}}\left\|v_{h}\right\|_{X_{1}} \\
\forall v & \in \widehat{T}_{h}, & \widehat{a}_{h}(v, v) & \geq \widehat{c}_{3}\|v\|_{X_{1}}^{2}, \\
\forall v_{h} & \in \widehat{X}_{h}, & \left\langle\mathcal{A} v_{h}, \mathcal{A} v_{h}\right\rangle_{h} & \leq \widehat{c}_{4}\left\|v_{h}\right\|_{X_{1}}^{2} \\
\forall \mu_{h} & \in \bar{M}_{h}, & \left\langle\mathcal{B} \mu_{h}, \mathcal{B} \mu_{h}\right\rangle_{h} & \leq \widehat{c}_{5}\left\|\mu_{h}\right\|_{X_{1}}^{2} \\
\frac{\widehat{c}_{3}}{2} & \geq \widehat{c}_{4}^{2} .
\end{array}
$$

Comme auparavant, pour avoir une écriture compacte, on introduit les formes bilinéaires suivantes : $B_{h}$ définie sur $Q_{h} \times Q_{h}$ par

$$
B_{h}\left(\left(u_{h}, \lambda_{h}\right),\left(v_{h}, \mu_{h}\right)\right)=a_{h}\left(u_{h}, v_{h}\right)+b_{h}\left(\lambda_{h}, v_{h}\right)+b_{h}\left(\mu_{h}, u_{h}\right)
$$

$\bar{B}_{h}$ définie sur $\bar{Q}_{h} \times \bar{Q}_{h}$ par

$$
\bar{B}_{h}\left(\left(u_{h}, \lambda_{h}\right),\left(v_{h}, \mu_{h}\right)\right)=\bar{a}_{h}\left(u_{h}, v_{h}\right)+\bar{b}_{h}\left(\lambda_{h}, v_{h}\right)+\bar{b}_{h}\left(\mu_{h}, u_{h}\right)
$$

et $\widehat{B}_{\alpha, h}$ définie sur $\widehat{Q}_{h} \times \widehat{Q}_{h}$ par

$$
\widehat{B}_{\alpha, h}\left(\left(u_{h}, \lambda_{h}\right),\left(v_{h}, \mu_{h}\right)\right)=\widehat{a}_{h}\left(u_{h}, v_{h}\right)+\widehat{b}_{h}\left(\lambda_{h}, v_{h}\right)+\widehat{b}_{h}\left(\mu_{h}, v_{h}\right)+\alpha\left\langle\mathcal{A} u_{h}+\mathcal{B} \lambda_{h}, \mathcal{A} u_{h}-\alpha \mathcal{B} \mu_{h}\right\rangle_{h},
$$

où $\alpha=-1,1$, et on définit la forme linéaire $\widehat{L}_{h}$ sur $\widehat{X}_{h} \times \widehat{M}_{h}$ par $\widehat{L}_{h}\left(\left(v_{h}, \mu_{h}\right)\right)=\widehat{l}_{h}\left(v_{h}\right)+\widehat{g}_{h}\left(\mu_{h}\right)$.

On considère le problème intermédiaire suivant :

$$
\left(\widehat{P}_{\alpha, h}\right)\left\{\begin{array}{l}
\text { trouver } \widehat{\mathcal{E}}_{h} \in \widehat{Q}_{h} \text { solution de }: \\
\widehat{B}_{\alpha, h}\left(\widehat{\mathcal{E}}_{h}, \widehat{V}_{h}\right)=\widehat{L}_{h}\left(\widehat{V}_{h}\right)-\bar{B}_{h}\left(U_{h}, \widehat{V}_{h}\right), \quad \forall \widehat{V}_{h} \in \widehat{Q}_{h}
\end{array}\right.
$$

où $U_{h}=\left(u_{h}, \lambda_{h}\right)$ et $\widehat{V}_{h}=\left(v_{h}, \mu_{h}\right) \in \widehat{X}_{h} \times \widehat{M}_{h}$.

Théorème 4. Si les relations (25-29) sont vérifiées, alors le problème ( $\left.\widehat{P}_{h}\right)$ admet une solution et une seule.

Preuve. Elle découle directement du corollaire 1, paragraphe 2.3.

Nous supposerons, comme c'est le cas dans l'analyse des estimations a posteriori hiérarchiques, que :

$\left(\mathrm{H}_{1}\right)$

$$
\left\|U-\bar{U}_{h}\right\|_{Q_{1}} \leq \beta^{\prime}\left\|U-U_{h}\right\|_{Q_{1}} \text { où } 0<\beta^{\prime}<1 \text { constante, }
$$

où $\|(v, \mu)\|_{Q_{1}}=\left(\|v\|_{X_{1}}^{2}+\|\mu\|_{M_{1}}^{2}\right)^{1 / 2}, U_{h}$ (resp. $\left.\bar{U}_{h}\right)$ sont les solutions du problème $\left(P_{h}\right)$ (resp. $\left.\left(\bar{P}_{h}\right)\right)$;

$\left(\mathrm{H}_{2}\right) \quad$ il existe une constante $\left.\gamma_{2} \in\right] 0,1\left[\right.$ (indépendante des dimensions des espaces $X_{h}, \widehat{X}_{h}, \bar{X}_{h}, M_{h}, \widehat{M}_{h}, \bar{M}_{h}$ ) telle que

$$
\forall V \in Q_{h}, \quad W \in \widehat{Q}_{h}:\left|(V, W)_{Q_{1}}\right| \leq \gamma_{2}\|V\|_{Q_{1}}\|W\|_{Q_{1}}
$$

où $(., .)_{Q_{1}}$ est le produit scalaire sur $Q_{1}=X_{1} \times M_{1}$. 
Remarques. La constante $\gamma_{2}$ est appelée constante de C.B.S. forte (voir [2]). Le résultat principal de ce paragraphe est le :

Théorème 5. Si U est solution de $(P), U_{h}$ solution de $\left(P_{h}\right), \widehat{\mathcal{E}}_{h}$ solution de $\left(\widehat{P}_{h}\right)$, alors, sous les conditions (21-24), (25-30) et les hypothèses $\left(\mathrm{H}_{1}\right)$ et $\left(\mathrm{H}_{2}\right)$, l'estimateur a posteriori $\widehat{\mathcal{E}}_{h}=\left(\widehat{u}_{h}, \widehat{\lambda}_{h}\right)$ vérifie les inégalités suivantes $(\alpha=-1,1)$ :

$$
\left\|\widehat{\mathcal{E}}_{h}\right\| \leq C_{1}\left\{\left\|U-U_{h}\right\|_{Q_{1}}+\sup _{W \in \widehat{Q}_{h},\|W\|=1}\left[\left(\widehat{L}_{h}(W)-\bar{L}_{h}(W)\right]\right\}\right.
$$

et

$$
\begin{aligned}
\left\|U-U_{h}\right\|_{Q_{1} \leq} \leq & C_{2}\left\{\widehat { C } \left(\left\|\left|\widehat{\mathcal{E}}_{h}\|\mid+\| \widehat{\lambda}_{h} \|_{M_{1}}\right)+\sup _{V \in Q_{h},\|\mid V\| \|=1}\left[\bar{L}_{h}(V)-\bar{B}_{h}\left(U_{h}, V\right)\right]\right.\right.\right. \\
& \left.+\sup _{W \in \widehat{Q}_{h},\|W\|=1}\left[\bar{L}_{h}(W)-\widehat{L}_{h}(W)\right]\right\}
\end{aligned}
$$

où :

$$
\begin{aligned}
& C_{1}=\sup \left(\frac{1}{\hat{\beta}}, \frac{\bar{C}}{\hat{\beta}}\left(1+\beta^{\prime}\right) \sup \left(1, \widehat{c}_{3}\right)\right) \\
& C_{2}=\frac{1}{\bar{\beta}\left(1-\beta^{\prime}\right) \sqrt{1-\gamma_{2}^{2}}} .
\end{aligned}
$$

Preuve. Commençons par démontrer la première inégalité. On a d'après l'hypothèse de saturation :

$$
\left(1-\beta^{\prime}\right)\left\|U-U_{h}\right\|_{Q_{1}} \leq\left\|\bar{U}_{h}-U_{h}\right\|_{Q_{1}}
$$

et

$$
\left\|\bar{U}_{h}-U_{h}\right\|_{Q_{1}} \leq\left(1+\beta^{\prime}\right)\left\|U-U_{h}\right\|_{Q_{1}} .
$$

Or,

$$
\begin{aligned}
\widehat{\beta} \| \mid\left\{\widehat{\mathcal{E}}_{h} \| \mid\right. & \leq \sup _{W \in \widehat{Q}_{h},\|\mid W\|=1} \widehat{B}_{\alpha, h}\left(\widehat{\mathcal{E}}_{h}, W\right) \\
& =\sup _{W \in \widehat{Q}_{h},\|W\|=1}\left\{\widehat{L}_{h}(W)-\bar{B}\left(U_{h}, W\right)\right\} \\
& =\sup _{W \in \widehat{Q}_{h},\|W\|=1}\left\{\widehat{L}_{h}(W)-\bar{B}\left(\bar{U}_{h}, W\right)+\bar{B}\left(\bar{U}_{h}-U_{h}, W\right)\right\} \\
& \leq \bar{C}\left\|\bar{U}_{h}-U_{h}\right\| \mid+\sup _{W \in \widehat{Q}_{h},\|W\|=1}\left\{\widehat{L}_{h}(W)-\bar{L}_{h}(W)\right\} \\
& \leq \bar{C} \sup \left\{1, \widehat{c}_{3}\right\}\left\|\bar{U}_{h}-U_{h}\right\|_{Q_{1}}+\sup _{W \in \widehat{Q}_{h},\|W\|=1}\left\{\widehat{L}_{h}(W)-\bar{L}_{h}(W)\right\} \\
& \leq \bar{C}\left(1+\beta^{\prime}\right) \sup \left\{1, \widehat{c}_{3}\right\}\left\|U-U_{h}\right\|_{Q_{1}}+\sup _{W \in \widehat{Q}_{h},\|W\|=1}\left\{\widehat{L}_{h}(W)-\bar{L}_{h}(W)\right\}
\end{aligned}
$$


Par suite,

$$
\left\|\widehat{\mathcal{E}}_{h}\right\| \mid \leq \frac{\bar{C}}{\widehat{\beta}}\left(1+\beta^{\prime}\right) \sup \left\{1, \widehat{c}_{3}\right\}\left\|U-U_{h}\right\|_{Q_{1}}+\frac{1}{\widehat{\beta}_{W \in \widehat{Q}_{h},\||W \||=1}} \sup _{\left.\widehat{L}_{h}(W)-\bar{L}_{h}(W)\right\}}
$$

Démontrons maintenant la deuxième inégalité. Remarquons tout d'abord que pour tout couple $(V, W) \in Q_{h} \times \widehat{Q}_{h}$ vérifiant $\|V+W\|_{Q_{1}}=1$ on a :

$$
1 \geq\left(1-\gamma_{2}^{2}\right)\|V\| \quad \text { et } \quad 1 \geq\left(1-\gamma_{2}^{2}\right)\|W\|
$$

D’une part,

$$
\begin{aligned}
\bar{\beta}\left\|\bar{U}_{h}-U_{h}\right\|_{Q_{1}} & \leq \sup _{\|\mid V+W\|=1} \bar{B}_{h}\left(\bar{U}_{h}-U_{h}, V+W\right) \\
& =\sup _{\|\mid V+W\|=1}\left\{\bar{L}_{h}(V)+\widehat{B}_{\alpha, h}\left(\widehat{\mathcal{E}}_{h}, W\right)-\bar{B}_{h}\left(U_{h}, V\right)+\bar{L}_{h}(W)-\widehat{L}_{h}(W)\right\} .
\end{aligned}
$$

D'autre part, pour tout $W=(v, \mu) \in \widehat{Q}_{h}$ tel que $\|W\|_{Q_{1}}=1$, on a :

$$
\begin{aligned}
\widehat{B}_{\alpha, h}\left(\widehat{\mathcal{E}}_{h}, W\right) & \leq\left(\left\|\widehat{\mathcal{E}}_{h}\right\|_{Q_{1}}+\left|\mathcal{A} \widehat{u}_{h}\right|_{h}+\left|\mathcal{B} \widehat{\lambda}_{h}\right|_{h}\right)\left(\left\|\widehat{W}_{h}\right\|_{Q_{1}}+|\mathcal{A} v|_{h}+|\mathcal{B} \mu|_{h}\right) \\
& \leq \widehat{C}\left(\left\|\widehat{\mathcal{E}}_{h}\right\| \mid+\left\|\widehat{\lambda}_{h}\right\|_{M_{1}}\right)\|\widehat{W}\|_{Q_{1}} \\
& \leq \frac{\widehat{C}}{\sqrt{1-\gamma_{2}^{2}}}\left(\left\|\widehat{\mathcal{E}}_{h}+\right\| \widehat{\lambda}_{h} \|_{M_{1}}\right) .
\end{aligned}
$$

Par suite,

$$
\begin{aligned}
\bar{\beta}\left\|\bar{U}_{h}-U_{h}\right\|_{Q_{1}} \leq & \frac{\widehat{C}}{\sqrt{1-\gamma_{2}^{2}}}\left\{\widehat{C}\left(\left\|\widehat{\mathcal{E}}_{h}\right\| \mid+\left\|\widehat{\lambda}_{h}\right\|_{M_{1}}\right)+\sup _{\|V\|_{Q_{1}}=1}\left(\bar{L}_{h}(V)-\bar{B}_{h}\left(U_{h}, V\right)\right)\right. \\
& \left.+\sup _{\|W\|_{Q_{1}}=1}\left(\bar{L}_{h}(W)-\widehat{L}_{h}(W)\right)\right\} .
\end{aligned}
$$

On en déduit alors que :

$$
\begin{aligned}
\overline{\| U}-U_{h} \|_{Q_{1}} & \leq \frac{1}{\bar{\beta}\left(1-\beta^{\prime}\right) \sqrt{1-\gamma_{2}^{2}}}\left\{\widehat{C}\left(\left\|\widehat{\mathcal{E}}_{h}\right\| \mid+\left\|\widehat{\lambda}_{h}\right\|_{M_{1}}\right)\right. \\
& \left.+\sup _{V \in Q_{h},\|V\| \|=1}\left(\bar{L}_{h}(V)-\bar{B}_{h}\left(U_{h}, V\right)\right)+\sup _{W \in \widehat{Q}_{h},\|W\| \mid=1}\left(\bar{L}_{h}(W)-\widehat{L}_{h}(W)\right)\right\} .
\end{aligned}
$$

Sous certaines hypothèses plus fortes, on peut obtenir des estimations a posteriori avec une norme plus naturelle. Plus précisément, si on considère la norme \|\|$_{Q_{1}, h}$ définie par :

$$
\|(v, \mu)\|_{Q_{1}, h}^{2}=\|v\|_{X_{1}}^{2}+\left\|\mu_{h}\right\|_{M_{1}}^{2}+|\mathcal{B} \mu|_{h}^{2}
$$


on a le

Théorème 6. Avec les mêmes hypothèses que le théorème 5, et si on suppose en plus que la forme $\widehat{b}_{h}(.,$. vérifie

$$
\forall \mu_{h} \in \widehat{M}_{h}, \quad \sup _{v_{h} \in \widehat{X}_{h}} \frac{\widehat{b}_{h}\left(\mu_{h}, v_{h}\right)}{\left\|v_{h}\right\|_{X_{1}}} \geq \widehat{c}_{4}\left\|\mu_{h}\right\|_{M_{1}}-\widehat{c}_{5}\left|\mathcal{B} \mu_{h}\right|_{h}
$$

alors l'estimateur a posteriori $\widehat{\mathcal{E}}_{h}$ vérifie les inégalités suivantes $(\alpha=-1,1)$ :

$$
\left\|\widehat{\mathcal{E}}_{h}\right\|_{Q_{1}, h} \leq C_{1}\left\{\left\|U-U_{h}\right\|_{Q_{1}}+\sup _{W \in \widehat{Q}_{h},\|W\|_{Q_{1}, h}=1}\left\{\left(\widehat{L}_{h}(W)-\bar{L}_{h}(W)\right\}\right\}\right.
$$

et

$$
\begin{aligned}
& \left\|U-U_{h}\right\|_{Q_{1}} \leq C_{2}\left\{\widehat{C}\left\|\widehat{\mathcal{E}}_{h}\right\|_{Q_{1}, h}+\sup _{V \in Q_{h},\|V\|_{Q_{1}, h}=1}\left[\left(\bar{L}_{h}(V)-\bar{B}_{h}\left(U_{h}, V\right)\right]\right.\right. \\
& \left.+\sup _{W \in \widehat{Q}_{h},\|W\|_{Q_{1, h}=1}}\left[\bar{L}_{h}(W)-\widehat{L}_{h}(W)\right]\right\}
\end{aligned}
$$

$o \grave{u}$

$$
\begin{gathered}
C_{1}=\frac{\bar{C}}{\hat{\beta}}\left(1+\beta^{\prime}\right) \\
C_{2}=\frac{1}{\bar{\beta}\left(1-\beta^{\prime}\right) \sqrt{1-\gamma_{2}^{2}}} .
\end{gathered}
$$

Preuve. En utilisant la remarque développée dans la section 2.5, la preuve est similaire à celle du théorème précédent.

\subsection{Autres cas}

Un cas très intéressant est celui où l'hypothèse (23) est vérifiée seulement pour la forme bilinéaire $b_{h}(.,$.$) ,$ i.e. les espaces $\left(\bar{X}_{h}, \bar{M}_{h}\right)$ ne sont pas fortement compatibles. Dans ce cas, le problème discret $\left(\bar{P}_{h}\right)$ devrait être remplacé par un problème stabilisé. Pour cela, on considère le problème augmenté suivant :

$$
\left\{\begin{array}{l}
\left(\bar{u}_{h}, \bar{\lambda}_{h}\right) \in \bar{X}_{h} \times \bar{M}_{h} \\
\bar{B}_{\bar{\alpha}, h}\left(\left(\bar{u}_{h}, \bar{\lambda}_{h}\right),\left(\bar{v}_{h}, \bar{\mu}_{h}\right)=\bar{L}_{\bar{\alpha}, h}\left(\bar{v}_{h}, \bar{\mu}_{h}\right), \quad \forall\left(\bar{v}_{h}, \bar{\mu}_{h}\right) \in \bar{X}_{h} \times \bar{M}_{h}\right.
\end{array}\right.
$$

où $\bar{B}_{\bar{\alpha}, h}$ et $\bar{L}_{\bar{\alpha}, h}$ sont respectivement la forme bilinéaire et la forme linéaire stabilisées, sur $\bar{X}_{h} \times \bar{M}_{h}$, avec $\bar{\alpha}$ comme facteur de stabilisation.

On considère le problème intermédiaire suivant :

$$
\left\{\begin{array}{l}
\widehat{\mathcal{E}}_{h} \in \widehat{Q}_{h} \\
\widehat{B}_{\widehat{\alpha}, h}\left(\widehat{\mathcal{E}}_{h}, \widehat{V}_{h}\right)=\widehat{L}_{\bar{\alpha}, h}\left(\widehat{V}_{h}\right)-\bar{B}_{\bar{\alpha}, h}\left(U_{h}, \widehat{V}_{h}\right), \quad \forall \widehat{V}_{h} \in \widehat{Q}_{h}
\end{array}\right.
$$


avec $(\bar{\alpha}, \widehat{\alpha}) \in\{-1,1\}^{2}$. Comme auparavant, on suppose que les hypothèses $\left(\mathrm{H}_{1}\right)$ et $\left(\mathrm{H}_{2}\right)$ sont vérifiées, et on suppose de plus que :

$$
\forall \mu_{h} \in \widehat{M}_{h} \quad \sup _{v_{h} \in \widehat{X}_{h}} \frac{\bar{b}_{h}\left(\mu_{h}, v_{h}\right)}{\left\|v_{h}\right\|_{X_{1}}} \geq c_{4}\left\|\mu_{h}\right\|_{M_{1}}-c_{5}\left|\mathcal{B} \mu_{h}\right|_{h}
$$

Dans ce cas, on a le même résultat que le théorème 5. Plus précisément :

$$
\left\|\left|\widehat{\mathcal{E}}_{h}\left\|\mid \leq C_{1}\right\| U-U_{h} \|_{Q_{1}, h}+\sup _{W \in \widehat{Q}_{h},\|W\| \mid \|=1}\left(\widehat{L}_{\bar{\alpha}, h}(W)-\bar{L}_{\bar{\alpha}, h}(W)\right)\right.\right.
$$

et

$$
\begin{aligned}
\left\|U-U_{h}\right\|_{Q_{1}, h} \leq & \frac{C}{\left(1-\beta^{\prime}\right) \sqrt{1-\gamma_{2}^{2}}}\left\{\left\|\widehat{\mathcal{E}}_{h}\right\| \mid+\left\|\widehat{\lambda}_{h}\right\|_{M_{1}}\right. \\
& +\sup _{V \in Q_{h},\|\mid V\| l=1}\left[\bar{L}_{\bar{\alpha}, h}(V)-\bar{B}_{\bar{\alpha}, h}\left(U_{h}, V\right)\right] \\
& \left.+\sup _{W \in \widehat{Q}_{h},\|W\| \mid=1}\left[\bar{L}_{\bar{\alpha}, h}(W)-\widehat{L}_{\bar{\alpha}, h}(W)\right]\right\} .
\end{aligned}
$$

Remarque. On a $\widehat{L}_{\bar{\alpha}, h}$ au lieu de $\bar{L}_{\bar{\alpha}, h}$ dans le problème intermédiaire.

\section{Applichtion}

Dans le présent paragraphe, on donne un exemple d'application, de construction d'un estimateur a posteriori hiérarchique pour le problème de Stokes stabilisé.

\subsection{Problème de Stokes}

On considère le problème de Stokes dans un ouvert, convexe polygonal et borné $\Omega$ de $\mathbb{R}^{2}$

$$
(P) \begin{cases}-\nu \Delta \underline{u}+\nabla p=f & \text { dans } \Omega \\ \operatorname{div} \underline{u}=0 & \text { dans } \Omega \\ \underline{u}=0 & \text { sur } \Gamma .\end{cases}
$$

La formulation variationnelle du problème $(P)$ est donnée par :

$$
\left\{\begin{array}{l}
\text { trouver }(\underline{u}, p) \quad \text { dans }\left(H_{0}^{1}(\Omega)\right)^{2} \times L_{0}^{2}(\Omega) \text { tels que : } \\
\forall \underline{v} \in H_{0}^{1}(\Omega)^{2}, \quad \nu \int_{\Omega} \nabla \underline{u} . \nabla \underline{v} \mathrm{~d} x-\int_{\Omega} \operatorname{div}(\underline{v}) p \mathrm{~d} x=\langle\underline{f}, \underline{v}\rangle \\
\forall q \in L_{0}^{2}(\Omega), \quad \int_{\Omega} \operatorname{div}(\underline{u}) q \mathrm{~d} x=0 .
\end{array}\right.
$$

La viscosité $\nu$ est réelle positive et la fonction $\underline{f}$ est supposée dans $\left(L^{2}(\Omega)\right)^{2}$. On pose

$$
a(\underline{u}, \underline{v})=\nu(\nabla \underline{u}, \nabla \underline{v})_{0, \Omega},
$$

et

$$
\begin{gathered}
b(\underline{v}, p)=-(p, \operatorname{div} \underline{v})_{0, \Omega}, \\
X=\left(H_{0}^{1}(\Omega)\right)^{2}, \quad M=L_{0}^{2}(\Omega)
\end{gathered}
$$


et les normes $\|\cdot\|_{X}$, et $\|\cdot\|_{M}$ sur $X$ et $M$ respectivement par :

$$
\begin{gathered}
\|\underline{u}\|_{X}=\|\nabla \underline{u}\|_{0, \Omega}=|\underline{u}|_{1, \Omega} \\
\|p\|_{M}=\|p\|_{0, \Omega} .
\end{gathered}
$$

Soit $\mathcal{T}_{h}$ une triangulation régulière de $\Omega$ en triangles. On considère les espaces

$$
X_{h}=\left\{\underline{v} \in\left(\mathcal{C}^{0}(\bar{\Omega})^{2} / \forall K \in \mathcal{T}_{h},\left.\underline{v}\right|_{K} \in\left(P_{1}+\operatorname{Vect}\left(\left\{\psi_{K}\right\}\right)\right)^{2}\right\} \cap\left(H_{0}^{1}(\Omega)\right)^{2}\right.
$$

où $\psi_{K}$ est la fonction bulle associée à l'élément $K$ et

$$
M_{h}=\left\{q \in \mathcal{C}^{0}(\bar{\Omega}) / \forall K \in \mathcal{T}_{h},\left.q\right|_{K} \in P_{1}(K) \cap L_{0}^{2}(K)\right\} .
$$

Le problème discret s'écrit :

$$
\left(P_{h}\right)\left\{\begin{array}{l}
\text { trouver }\left(\underline{u}_{h}, p_{h}\right) \in X_{h} \times M_{h} \text { tel que : } \\
\forall \underline{v}_{h} \in X_{h}, \quad a\left(\underline{u}_{h}, \underline{v}_{h}\right)+b\left(\underline{v}_{h}, p_{h}\right)=\left\langle f, \underline{v}_{h}\right\rangle \\
\forall q_{h} \in M_{h}, \quad b\left(\underline{u}_{h}, q_{h}\right)=0 .
\end{array}\right.
$$

On définit en plus les espaces :

$$
\begin{aligned}
\bar{X}_{h} & =\left\{\underline{v} \in\left(\mathcal{C}^{0}(\bar{\Omega})^{2} /\left.\underline{v}\right|_{K} \in\left(P_{2}+\operatorname{Vect}\left(\left\{\psi_{K}\right\}\right)\right)^{2}\right\} \cap\left(H_{0}^{1}(\Omega)\right)^{2}\right. \\
\bar{M}_{h} & =\left\{q \in \mathcal{C}^{0}(\bar{\Omega}) /\left.q\right|_{K} \in P_{2}(K)\right\} \cap L_{0}^{2}(\Omega) \\
\widehat{X}_{h} & =\left\{\underline{v} \in\left(\mathcal{C}^{0}(\bar{\Omega})^{2} /\left.\underline{v}\right|_{K} \in\left(P_{2}(K)\right)^{2} \text { et } \underline{v}=0 \text { aux sommets de } K\right\} \cap\left(H_{0}^{1}(\Omega)\right)^{2}\right. \\
\widehat{M}_{h} & =\left\{q \in \mathcal{C}^{2}(\Omega) /\left.q\right|_{K} \in P_{2}(K) \text { et } q=0 \text { aux sommets de } K\right\} \cap L_{0}^{2}(\Omega) .
\end{aligned}
$$

On vérifie facilement que :

$$
\begin{aligned}
\bar{X}_{h} & =X_{h} \oplus \widehat{X}_{h} \\
\bar{M}_{h} & =M_{h} \oplus \widehat{M}_{h} .
\end{aligned}
$$

Remarque. Vu que $\left\{\underline{v} \in\left(\mathcal{C}^{0}(\bar{\Omega})^{2} /\left.\underline{v}\right|_{K} \in\left(P_{2}(K)\right)^{2}\right\} \subset \bar{X}_{h}\right.$ et $\bar{M}_{h} \subset \mathcal{C}^{0}(\Omega)$, le lemme de Franca-HughesStenberg [11] (voir paragraphe 2, lemme 3) nous permet d'affirmer que l'hypothèse (15) est vérifiée.

Proposition 2. Les espaces $\left(X_{h}, M_{h}\right)$ sont fortement compatibles.

Les espaces $\bar{X}_{h}, \bar{M}_{h}$ ne sont pas fortement compatibles, cela suggère l'utilisation d'une formulation stabilisée pour discrétiser le problème de Stokes dans ce cas.

Pour appliquer le théorie abstraite développée dans le deuxième paragraphe, nous avons besoin de définir les opérateurs et les espaces suivants :

$$
\begin{aligned}
\mathcal{A}: X_{1} & \longrightarrow H_{1}, \\
\underline{v} & \longmapsto\left(\left(-\left.\Delta \underline{v}\right|_{K}\right)\right)_{K \in \mathcal{T}_{h}},
\end{aligned}
$$

et

$$
\begin{aligned}
\mathcal{B}: M_{1} & \longrightarrow H_{1}, \\
p & \longmapsto\left(\nabla\left(\left.p\right|_{K}\right)\right)_{K \in \mathcal{T}_{h}},
\end{aligned}
$$


où

et

$$
X_{1}=X=\left(H_{0}^{1}(\Omega)\right)^{2}, M_{1}=M=L_{0}^{2}(\Omega)
$$

$$
H_{1}=\prod_{K \in \mathcal{T}_{h}}\left(H^{-1}(K)\right)^{2}
$$

On définit l'espace

$$
H=\prod_{K \in \mathcal{T}_{h}}\left(L^{2}(K)\right)^{2}
$$

Tout d'abord, remarquons que si $(\underline{u}, p)$ est la solution de Stokes, on a :

$$
\mathcal{A} \underline{u}+\mathcal{B} p=\underline{f} \quad \text { dans } H .
$$

On considère la forme bilinéaire $\langle., .\rangle_{h}$ définie sur $\left(L^{2}(\Omega)\right)^{2}$ par :

$$
\langle p, q\rangle_{h}=\sum_{K \in \mathcal{T}_{h}} \delta_{K} h_{K}^{2}\langle p, q\rangle_{0, K}
$$

où les $\left(\delta_{K}\right)_{K \in \mathcal{T}_{h}}$ sont des paramètres réels positifs. Pour avoir une écriture compacte des problèmes discrets, nous allons introduire quelques notations.

On définit la forme bilinéaire $B: Q^{2} \longrightarrow \mathbb{R}$ par $B((\underline{u}, p) ;(\underline{v}, q))=a(\underline{u}, \underline{v})+b(p, \underline{v})+b(q, \underline{u})$, les formes bilinéaires $\widetilde{B}_{\widetilde{\alpha}, h}: \widetilde{Q}_{h} \times \widetilde{Q}_{h} \longrightarrow \mathbb{R}$ par :

$$
\widetilde{B}_{\widetilde{\alpha}, h}\left(\left(\underline{u}_{h}, p_{h}\right) ;\left(\underline{v}_{h}, q_{h}\right)\right)=B\left(\left(\underline{u}_{h}, p_{h}\right) ;\left(\underline{v}_{h}, q_{h}\right)\right)+\widetilde{\alpha}\left\langle\mathcal{A} \underline{u}_{h}+\mathcal{B} p_{h}, \mathcal{A} \underline{v}_{h}-\widetilde{\alpha} \mathcal{B} q_{h}\right\rangle_{h}
$$

et les formes linéaires $\widetilde{L}_{\bar{\alpha}, h}: \widetilde{Q}_{h} \longrightarrow \mathbb{R}$ par $\widetilde{L}_{\bar{\alpha}, h}\left(\left(\underline{v}_{h}, q_{h}\right)\right)=\int_{\Omega} \underline{f} \underline{v} \mathrm{~d} x+\bar{\alpha}\left\langle\underline{f}, \mathcal{A} \underline{v}_{h}-\underline{\alpha} \mathcal{B} q_{h}\right\rangle_{h}$ où $\backsim=\wedge,-$ et $\widetilde{\alpha} \in\{-1,1\}$. On est en mesure de définir alors le problème auxiliaire :

$$
\left(\widehat{P}_{h}\right)\left\{\begin{array}{l}
\text { trouver } \widehat{\mathcal{E}}_{h} \in \widehat{Q}_{h} \text { tel que : } \\
\widehat{B}_{\widehat{\alpha}, h}\left(\widehat{\mathcal{E}}_{h}, \widehat{V}_{h}\right)=\widehat{L}_{\bar{\alpha}, h}\left(\widehat{V}_{h}\right)-B_{\bar{\alpha}, h}\left(U_{h}, \widehat{V}_{h}\right) \quad \forall \widehat{V}_{h} \in \widehat{Q}_{h}
\end{array}\right.
$$

On a la

Proposition 3. Si $U=(\underline{u}, p)$ est la solution du problème $(P), \widehat{\mathcal{E}}_{h}=\left(\widehat{u}_{h}, \widehat{p}_{h}\right)$ est la solution du problème $\left(\widehat{P}_{h}\right)$, alors sous les hypothèses $\left(\mathrm{H}_{1}\right)$ et $\left(\mathrm{H}_{2}\right)$. Si $\left(\delta_{K}\right)_{K \in \mathcal{T}_{h}}$ sont assez petits, on a, pour $\widetilde{\alpha}=1$ ou -1 , les estimations suivantes :

$$
\left|\underline{u}_{h}\right|_{1, \Omega}+\left(\sum_{K \in \mathcal{T}_{h}} h_{K}^{2}\left|\nabla \widehat{p}_{h}\right|_{0, K}^{2}\right)^{\frac{1}{2}} \leq C_{1}\left(\left|\underline{u}-\underline{u}_{h}\right|_{1, \Omega}+\left|p-p_{h}\right|_{0, \Omega}\right)
$$

et

$$
\left|\underline{u}-\underline{u}_{h}\right|_{1, \Omega}+\left|p-p_{h}\right|_{0, \Omega} \leq \frac{C_{2}}{\left(1-\beta^{\prime}\right) \sqrt{1-\gamma^{2}}}\left\{\left|\underline{\widehat{u}}_{h}\right|_{1, \Omega}+\left\|\widehat{p}_{h}\right\|_{0, \Omega}\left(\sum_{K \in \mathcal{T}_{h}} h_{K}^{2}\left\|\nabla \widehat{p}_{h}\right\|_{0, K}^{2}\right)^{\frac{1}{2}}\right\} .
$$

Preuve. Pour pouvoir appliquer le théorème 5, nous avons besoin de vérifier les conditions (21-30). D'une part, il est évident que $(21,22,25-27)$ sont vérifiées. D'autre part, en utilisant la proposition 2 et les résultats classiques sur les formulations mixtes pour les équations de Stokes; on vérifie facilement que (24) est satisfaite. 
Les conditions $(28,29)$ sont les inégalités inverses "locales" (voir [9]). L’hypothèse (30) est vérifiée dès que les $\left(\delta_{K}\right)_{K \in \mathcal{T}_{h}}$ sont assez petits.

L'hypothèse (36) est bien vérifiée, car $\widehat{M}_{h} \subset \mathcal{C}^{0}(\bar{\Omega})$.

Nous sommes en mesure d'appliquer le théorème 5 . Par suite, on a le résultat.

\section{Remarques.}

1. Nous n'avons pas besoin d'uniforme régularité de la triangulation, car les seules inégalités inverses utilisées sont locales (sur chaque triangle $\mathrm{K}$ de $\mathcal{T}_{h}$ ).

2. Il est facile de montrer qu'il existe deux constantes strictement positives $C_{1}, C_{2}$, indépendantes de $h$, telles que pour tout $\widehat{p}_{h} \in \widehat{X}_{h}$, on a :

$$
\forall K \in \mathcal{T}_{h}, \quad C_{1}\left|\widehat{p}_{h}\right|_{0, K} \leq\left|\widehat{p}_{h}\right|_{1, K} h_{K} \leq C_{2}\left|\widehat{p}_{h}\right|_{0, K} .
$$

Par suite, on peut aussi considérer $\mathcal{E}_{h}=\left(\left|\widehat{u}_{h}\right|_{0, \Omega}^{2}+\left|\widehat{p}_{h}\right|_{0, \Omega}^{2}\right)^{\frac{1}{2}}$ comme estimateur a posteriori dans ce cas.

\section{BIBLIOGRAPHIE}

[1] B. Achchab, A. Agouzal, J. Baranger et J.F. Maitre, Estimateur d'erreur a posteriori hiérarchique. Application aux éléments finis mixtes. Numer. Math. 80 (1998) 159-179.

[2] B. Achchab et J.F. Maitre, Estimate of the constant in two strengthened C.B.S inequalities for F.E.M. system of the 2D elasticity. Application to multilevel methods and a posteriori error estimators. Numerical linear algebra with applications $\mathbf{1}$ (1995) 1-13.

[3] A. Agouzal, Analyse numérique des méthodes de décomposions de domaines. Méthodes des domaines fictifs. Thèse de doctorat, Université de Pau, France (1994).

[4] R.E. Bank et R.K. Smith, A posteriori error estimates based on hierarchical bases. SIAM J. Numer. Anal. 30 (1993) $921-935$.

[5] R.E. Bank et A. Weiser, Some a posteriori error estimators for elliptic partial differential equations. Math. Comp. 44 (1985) $283-301$.

[6] H.J.C. Barbosa et T.J.R. Hughes, The finite element method with Lagrange multipliers on the boundary: circumventing the Babuska-Brezzi condition. Comput. Meth. Appl. Eng. 85 (1992) 109-128.

[7] H.J.C. Barbosa et T.J.R. Hughes, Boundary Lagrange multipliers in finite element methods: error analysis in naturel norms. Numer. Math. 62 (1992) 1-15.

[8] F. Brezzi et M. Fortin, Mixed and Hybrid Finite Element Methods. Springer Series in Computational Mathematics 15 SpringerVerlag (1991).

[9] P.G. Ciarlet, The Finite Element for Elliptic Problems. North Holland, Amsterdam (1978).

[10] L.P. Franca, S. Frey et T.J.R. Hughes, Stabilized finite element methods: 1. Application to the advective-diffusive model. Comp. Methods Appl. Mech. Eng. 95 (1992) 253-276.

[11] L.P. Franca, T.J.R. Hughes et R. Stenberg, Stabilized finite element methods for the Stokes problem. Incompressible Computational Fluid Dynamics-Trends and Advances (à paraître).

[12] P.A. Raviart et J.M. Thomas, A mixed finite element method for second order elliptic problems, in Mathematical aspects of the finite Element Method, Galligani et Magenes Eds., Springer-Verlag (1977) 292-315.

[13] J.E. Roberts et J.M. Thomas, Mixed and hybrid methods, in Handbook of Numerical Analysis, P.G. Ciarlet et J.L. Lions Eds., Vol. 2, Part 1, North-Holland, Amsterdam (1989).

[14] J.M. Thomas, Sur l'analyse numérique des méthodes d'éléments finis hybrides et mixtes. Thèse d'état, Université Pierre et Marie Curie, France (1977). 